\title{
Première partie, introduction: De la possibilité de connaître le Tout
}

\author{
Gérard Bensussan
}

Le commentaire de cette l'Einleitung du Stern, vingt pages dans lédition de référence ${ }^{4}$, fera ici l'objet d'une lecture «carrée ». "Carrée » signifie exactement cadrée entre les quatre coins que l'on peut discerner dans ce texte.

Les deux premiers sont opératifs (car ils commandent le Bris) :

-le Tout comme mensonge ;

-l'angoisse de mort comme vérité.

Les deux derniers sont constructifs (car ils commandent l'horizon de la Pensée Nouvelle) :

-la métaphore de la fresque et du tableau ;

-le maintien (Bewahrung) et l'avération (Bewährung).

Notre commentaire les emmêlera afin de restituer le tissu singulier et précis de l'Einleitung.

${ }^{4}$ GS II.

How to cite this book chapter:

Bensussan, G. 2021. Première partie, introduction: De la possibilité de connaître le Tout. In: Brasser, M., Bojanić, P. and Ciglia, F. P. (eds.) The Star for Beginners: Introductions to the Magnum Opus of Franz Rosenzweig. Pp. 11-21. London: Ubiquity Press. DOI: https://doi.org/10.5334/bco.c. License: CC-BY 


\section{Le Tout comme mensonge ${ }^{5}$}

Principe de départ et salubrité de la pensée : il convient de ne pas «être dupe " de la philosophie (S.4), selon une expression d'Emmanuel Levinas, et, surtout, de «son idée du Tout » dont la brume bleue et métaphysique enveloppe le terrestre, l'immanent, le matériel. Contre le " coucouville-les-nuées " (Aristophane) et les vapeurs de la philosophie, Rosenzweig s’affirme sans hésiter "matérialiste $»^{6}$ et associe son matérialisme à la révélation: « la révélation sauve la matière de la décomposition (Zersetzung) que lui impose l'idéalisme autant que le paganisme, elle lui redonne ses droits contre l'esprit, en-dedans de l'esprit, à l'âme contre l'esprit, et encore au temps contre l'atemporalité, à l'espace contre le concept, etc. [....] A propos de la "chair» : quelle force d'effectivité par rapport à Platon! $»^{7}$. Ce matérialisme de la révélation se déploie dès l'entrée en scène de la philosophie dans les premières pages du Stern selon un argument implacable et lapidaire. Premièrement, il convient de sérieusement méditer le statut traditionnel de "pensée de la connaissance une et universelle du Tout» de la philosophie (das eine und allgemeine Erkennen des All, 5) -tel est dailleurs le titre de l'Einleitung dans son ensemble, "Über die Möglichkeit, das All zu erkennen ». Cette tradition prévaut de Thalès ou Parménide à Hegel : le Tout sera déterminé en tant qu'il se comprend lui-même « sans reste " (restlos, 6). C'est évidemment Hegel, en tant qu'il achève et accomplit ce statut gnoséologique, qu'il s'agit ici de pleinement com-prendre pour mieux le déstructurer. Selon lui, l'autoprésentation de la pensée comme pensée du Tout s'incluant elle-même dans son automouvement systématisé va jusqu’à inclure la Révélation, en tant que représentation, dans le Tout de l'automouvement du concept. C'est cette circularité qui emporte la conclusion selon laquelle il faut tenir ce Tout pour un «mensonge », comme le mensonge de tous les mensonges (Lüge, Lügen, 5).

Pourquoi? Le présupposé réductible à la vieille proposition parménidienne, "être et penser, c'est le même ", a l'avantage de défaire d'emblée toute contre-proposition au nom de l'impossibilité pour le "sujet Tout " (Subjekt Alles, 13) de ne pas recevoir un prédicat parfaitement univoque (ein eindeutiges Prädikat, 13). : « une révolte contre la totalité du monde signifierait une négation de l'unité de la pensée » (ein Aufstand gegen die Allheit der Welt (bedeutet) zugleich eine Leugnung der Einheit des Denkens, 13). Autour de cet argument-massue, le gant est jeté à l'honorable confrérie des enfants de Parménide, de l'Ionie à Iéna (13). C'est en quelque sorte l'infalsibilité du présupposé qui en signale le «mensonge ». Au fond, le vrai, c'est bel et bien le Tout. Et ce constat (qui emporte un Aufstand!) est consacré et définitivement sanctionné par Hegel, lequel montre que le Tout, c'est son mouvement même, le Tout qui ne cesse jamais de se totaliser. L'Einleitung comporte une petite esquisse d'histoire

\footnotetext{
${ }^{5}$ Ibid., 5. Dans la suite, la pagination du Stern est intégrée au texte.

6 " Ich als Materialist », in GS I, 843.

${ }^{7}$ GS I, 559.
} 
de la philosophie qui aboutit à la philosophie de "notre temps " (unsere Zeit, 13). Cette philosophie de notre temps, Rosenzweig l'appelle, ailleurs, dans une lettre du 5 septembre 1916 à E. Rosenstock ${ }^{8}$, philosophie de la « forme du monde ", c'est-à-dire de la contingence pure et simple, du nun einmal so sein du monde. Le mensonge, c'est donc d'abord l'Unwahr du Tout qui annule cette contingence et se constitue en piège logique : la pensée doit prendre garde à ne pas s'y laisser enfermer si elle veut demeurer ce que Rosenzweig nomme notre "patrie» (Heimat, 15). Il y faut l'invention d'une figure concurrente du Tout omni-englobant (allumschliessend, 14). Cette figure, que Rosenzweig appelle dans l'Einleitung le Tout excluant (ausschliessend, 14), permettrait de laisser jouer l'imbrication des multiplicités Dieu Monde Homme pour déjouer l'engrenage fatal du Tout. Il ne s'agit pas d'une autre figure du Tout, d'un autre Tout, mais véritablement d'un infini non clos, d'une unicité expressive où s'articulent infiniment des multiplicités qu'on peut bien appeler des "touts », comme les tableaux ou les images de notre pensée, inégaux, discontinus, figurés, fragmentés, disposés sur un mur nu, indéterminé et matériel, où ils seraient suspendus.

\section{La fresque et le tableau (14)}

Pour Rosenzweig, tout se serait passé dans l'histoire de la métaphysique, de l'Ionie à Iéna, comme si le mur avait toujours été peint à fresques (al fresco). Lêtre (le mur) et la pensée (l'image, le tableau : suspendus ? dessinés ?) font Un, au sens le plus fort. On ne pourrait même pas les distinguer. Tel est le tout incluant, inclusif (ein allumschliessendes All, 14) : en fait tout un univers. On pourrait même dire qu'on a à faire, avec l'idéalisme, en fait d'univers, à un véritable trompe-l'oeil. On connaît l'anecdote rapportée par Pline l'Ancien : Zeuxis avait peint des raisins tellement parfaits que des oiseaux, trompés par une exécution si fidèle, se seraient jetés sur eux pour les consommer. Voilà bien l'idéalisme : il nous donne l'illusion de l'unité, illusion bienfaisante, consolante, esthétiquement aussi gratifiante qu'une belle fresque ou qu'un magnifique trompe-l'œil. Et cette jouissance ne cesse nullement avec le savoir de l'illusion comme illusion. Peut-être même s'accroît-elle encore par ce biais pervers.

Telle a longtemps été la situation historique de la pensée en Occident. Désormais au contraire, nous dit Rosenzweig, il faut tenir l'image, le tableau suspendu au mur, c'est-à-dire tel ou tel tableau, telle ou telle image, donc une singularité, pour un "tout excluant ", une unicité incluse (ein eingeschlossenes Eins, 14). Ceci requiert que soit pensé un rapport multiversel entre un support et une multiplicité infinie en droit. La pensée (le tableau dans la métaphore rosenzweigienne) n'est pas le moins du monde niée ou méprisée. Au contraire, elle est exhaussée dans sa singularité, dans le "point de vue » qu'elle propose dans les modes de philosopher " après Hegel » (117). Cette expression, le " point de

${ }^{8}$ GS I, 222. 
vue ", les " philosophes du point de vue », convient particulièrement et elle entre en congruence avec la métaphore de la fresque et de l'image. Un tableau ouvre une vue, il détache une vision dans le mur lui-même, à même son uniformité. Ce qui sengage nécessairement dans la distinction entre le mur et l'image qui y est accrochée (et qu'on peut décrocher), c'est l'exploration du rapport entre une unité et une multiplicité, entre une unité elle-même disséminée (ou cassée en trois " unités » Dieu Monde Homme) et une infinité de "patries ", c'est-à-dire d'images à chaque fois uniques et valant pour elles-mêmes de mille façons. Il s'agit donc, et tel est l'effet de la métaphore de la fresque et du tableau, de travailler à dégager les pensées ou les œuvres de toute totalisation, de toute universalisation, de toute contextualisation -pour les restituer à leur unicité inclusive et donc, par là même, exclusive.

Il y a toujours un au-delà du tableau : c'est le mur. Mais cet au-delà nen supprime nullement l'unicité. Il en forme bien plutôt la condition. Les rapports entre les deux, le mur et les images, relèvent alors d'une attention philosophique continue à la multiplicité inenglobable, aux restes de la totalisation du tout, à la non-identité originaire sur laquelle Rosenzweig insiste beaucoup dans ces pages (eine innere Nichtidentität, 14).

Au piège logique du Tout, soit à la confusion (Verwechslung, 17) entre le mur et le tableau qui est à «craindre " (fürchten), à son caractère de mensonge en trompe l'oeil, s'ajoute une autre détermination, un autre aspect du mensonge. Le mensonge du Tout est également, et même foncièrement, mensonger du point de vue moral, ou, beaucoup plus précisément, du point de vue de l'existence. Le Tout est mensonger parce qu'il dénie la finitude de l'existence, l'angoisse de mort des sujets mortels. Dans le Tout, rien ne meurt, un Tout, ça ne meurt pas (ein All würde nicht sterben und im All stürbe nichts, 4), cest sa fonction la plus évidente, sa force d'illusion vitale. Le trompe-l’eil est aussi un trompe-la-mort. Il y a une sorte de jeu redoutable et funeste entre le Tout et le Rien. Le tout « apaise » (beruhigt) et le rien est " paresseux » (träge) $)^{9}$. A la connaissance universelle du Tout et du Néant universels, Rosenzweig n’a cessé d’opposer une véritable philosophie du Quelque-Chose, non seulement dans les passages bien connus de l'Etoile, mais aussi dans toutes sortes de notations dont fourmille en particulier sa Correspondance, par exemple à propos de choses très empiriques et contingentes, le respect des mitzvot ou encore l'observance du chab$b a t^{10}$. Quelque chose, faire quelque chose, est toujours préférable à la ruineuse alternative du tout ou rien, à condition de ne pas négliger son absoluité : le quelque-chose est radicalement distinct d'un quelconque « juste-milieu».

${ }^{9}$ Lettre à R. Hallo du 27 mars 1922, GS I, 763.

${ }^{10}$ Ibid., 886. Rosenzweig conseille à Hallo, qui se demande s'il doit et ce qu'il doit faire pour observer le chabbat, d'inviter au moins quelques amis le vendredi soir, ce qui serait déjà quelque chose. 


\section{L'angoisse de mort comme "vérité "}

Le quelque-chose qui fait exception au Tout et au Rien, c'est le quelque-chose qui meurt. C'est par sa mort, qui nest pas Rien, qui enlève Tout, que le sujet existant, le Dasein créé est ce qu'il est. Son quelque chose d'irréductible, cest sa peur de la mort. Cela, la philosophie, l'idéalisme, le nie. Et la voie royale de cette négation, c'est le Tout. Il y a bien sûr un bénéfice secondaire de cette crainte niée, de cette angoisse subsumée dans une totalité où elle prend "place " (comme si la mort et la peur de la mort pouvaient se tenir à une place à côté d'une autre place !). Avant de statuer sur ce bénéfice secondaire, à savoir l'Erkennen, il faut sarrêter sur ce qu'on pourrait appeler l'effet de vérité de la mort, premier mot de l'arc qui se clôt « sur la vie », à la toute fin de l’ouvre, via «l'amour », arc métaphysique ou contre-métaphysique de la mort et de l'amour, où s'entrevoit peut-être une réminiscence de Schopenhauer -pour des conclusions radicalement opposées à celle du Welt als Wille und Vorstellung. Cet effet de vérité est d’abord un effet de choc. Celui-ci, très notable dans les premières pages, est sans doute calculé, selon une rhétorique, c'est-à-dire une stratégie discursive, qui peut faire penser au Sermon sur la mort de Bossuet sous la rubrique " ouvrir un tombeau». Ajoutons que la comparaison s'arrête là. Pour Bossuet en effet, l'homme, « en tant qu'il passe », est « méprisable » et, à ce titre, lauteur du Sermon sur la mort fait partie de l'honorable confrérie de ceux, philosophes d'une part, théologiens de lautre, qui distillent leur brume bleue, leur opium aux noms divers : Dieu ou bien le Tout, ou encore l'Esprit, c'est du pareil au même, soit ce que Schopenhauer nomme, au complément 41 du Welt als Wille und Vorstellung un puissant « antidote contre la certitude de la mort ». Contrairement à Bossuet, donc, pour Rosenzweig, «l'estimable», c'est l'existence, soit précisément l'homme "en tant qu'il passe » et trépasse.

L'Etoile de la rédemption souvre donc sur un effet de vérité prémédité, un choc mis en scène, une dramaturgie qui voit s'affronter deux protagonistes. D'un côté le Je vivant, irréductible, inconvertible en quoi que ce soit, vivant-contre jusquà ce qu'il meurt, terrifié, hurlant d’angoisse Je Je Je, tout entier corps. De l'autre la Philosophie, portraiturée en pied dans une sorte d'allégorie : une grande consolatrice, compatissante mais menteuse, sourire vide, air béat, indifférente et ayant l'air de se moquer, index pointé vers un au-delà du champ de bataille où le Je n’a qu'une peur, celle dêtre réduit à un ça (nur ein Es, 3). Ce qui suscite l'angoisse du Je, c'est qu'il se voit en ça, en troisième personne, en cadavre. Une brève remarque ici : pour comprendre jusquau bout et dans leur « logique " propre ces lignes qui ne sont pas le fin mot de l'Etoile sur la mort, il faudrait pouvoir souvrir déjà sur la révélation comme cette instance où la créature advient à son statut parlé de Je. La mort remet en question la révélation elle-même, en quelque sorte, en revenant en amont de la création, elle est une décréation retotalisante (304), tout comme l'existence est une révélation détotalisante. 
La Philosophie, dans l'allégorie où elle se pose dans les premières pages de l'Etoile, nous sermonne et nous assure que la mort n'est rien. Or la mort n'est pas rien, elle n'est pas ce rien dont on ne peut rien savoir, ce rien qui ne serait qu'un rien du savoir, justement (pour « l'idéalisme », bien sûr, le rien du savoir, c'est : rien du tout). Or la mort n'est pas du côté du néant ainsi déterminé, elle est bien quelque chose (5). Le grand présupposé de l'idéalisme (cette question du présupposé, du dunkle Voraussetzung (5), est absolument centrale), c'est que : 1. seul le pensable peut s'articuler en totalité ; 2 . seul le tout est pensable dans la mesure où la multiplicité qu'il englobe totalement peut se réduire à l'Un. Ceci, que Rosenzweig pointe comme le " grand présupposé », constitue le geste même, si l'on peut dire, de la pensée idéaliste, laquelle commence par réduire le multiple afin de le fonder ensuite en unitotalité. Hegel, le véritable penseur du "Tout où rien ne meurt », est évidemment le principal incriminé, sur ce point du « rien », de l' « ineffectif » de la mort : «Der Tod, wenn wir jene Unwirklichkeit so nennen wollen, ist das Furchtbarste, und das Tote fest zu halten, was die grösste Kraft erfordert. [...] Aber nicht das Leben, das sich vor dem Tode scheut und von der Verwüstung rein bewahrt, sondern das ihn erträgt, und in ihm erhält, ist das Leben des Geistes $"{ }^{11}$. Hegel pense le deuil et la douleur de la conscience comme une mort qui vient vivifier l'Esprit, la vie de l'Esprit. Pour lui, la mort est une extase négative: en se niant dans la différence, l'Esprit nie la différence en soi-même et la pose pour soi. La vie est alors le résultat substantiel de la négation surmontée, en-soi-pour-soi, et l'hégélianisme une sorte de stoïcisme christianisé où l'Esprit inscrit sa transcendance dans les plis de l'immanence. Ceci correspond exactement à la fonction du spéculatif telle que la détermine Rosenzweig -fonction de " protection » face à la mort (Schutz, 16), de déni et de forclusion ou encore, selon le mot de Jacques Derrida dans Glas, d' " amortissement » de la mort.

Seul le suicide pourrait à l'extrême rigueur constituer la forme sous laquelle la philosophie serait éventuellement à même d'envisager la mort. Lallusion au suicide, prudente, se trouve à la page 4 où la fameuse nuit de Leipzig de juillet 1913, la nuit de la tentation du suicide, est évoquée, via un mot (Phiole) emprunté à un vers du premier Faust: "Ich grüsse dich, du einzige Phiole ! / Die ich mit Andacht nun herunterhole ${ }^{12}$. Un an avant le début de la rédaction du Stern, le 13 août 1917, Rosenzweig revient encore sur cette nuit du suicide évité, dans une lettre à Eugen Rosenstock : « im übrigen war ich einfach auf den Mund geschlagen, noch viel zu nahe jenem völligen vis-a-vis du rien mit dem ich an jenem Morgen nach der Nacht in mein Zimmer gekommen war und meinen Browning 〈6,35〉 aus der Schreibtischschublade nahm ${ }^{13}$. Dans les premières pages du Stern, le « vis-à-vis du rien » est ainsi remémoré, douloureusement,

${ }^{11}$ G.W.F. Hegel, Phänomenologie des Geistes, Hamburg 2006, 26.

12 Johann Wolfgang von Goethe, Faust. Der Tragödie erster Teil [1829], Hg. Albert Schöne, Frankfurt a.M., 43.

13 GB, 22. 
au détour d'un développement sur l'angoisse de mort du combattant dans les tranchées du front -le « Browning 6.35 » ayant cédé la place à la fiole de poison.

Même si elle n'est pas sans importance, la question du suicide doit être ici mise de côté. Car ce qui se joue dans ces pages, c'est tout autre chose. Pour Rosenzweig, si l'Esprit peut regarder la mort en face, si la vie de l'Esprit se nourrit de son face à face avec la mort, comme l'écrit Hegel, alors c'est que cette mort est une pseudo-mort, un mensonge figuré et dissimulé sous les innombrables formes du Spéculatif : aliénation, scission, devenir-autre de l'Esprit, négativité. Pour Hegel, la mort, le mort, c'est la séparation, c'est le séparé. Pour Rosenzweig, au contraire, la mort c'est l'unité ultime, la retotalisation finale dans le cadavre. Ce que Rosenzweig détermine dans l'Einleitung comme Je (Ich), c'est l'anti-Esprit, si on peut ainsi dire, à savoir l'irréductible, l'inenglobable, l'inconvertible, brut et sauvage, sans devenir-autre ni Aufhebung. On songera ici à Kierkegaard qui disait : le système hégélien, dans l'auto-engendrement de sa vérité, peut parfaitement tout embrasser, tout totaliser, sauf Moi dès lors que je m’y refuse. Dans le paragraphe intitulé Kierkegaard (8), Rosenzweig se réapproprie la sentence afin de situer le sens du cri de la créature saisie d’effroi qui ouvre ces premières pages, tout en inaugurant la perspective, issue de l'angoisse de mort, d'une véritable métaphysique du nom propre : " denn mochte auch alles an ihm /dem Bewusstsein/ins Allgemeine zu überstezen sein - die Behaftetheit mit Vor- und Zunamen, das Eigene...blieb übrig, und gerade auf dies Eigene kam es " (8).

Une dernière précision résulte clairement de ce qui précède : ce n'est pas à la mort même que le Je doit faire face, c'est à l'angoisse de mort. Dans le premier cas, l'alternative oscillerait entre le suicide et la spéculation; dans le second cas, c'est de l'existence qu'il y va. Rosenzweig considère l'angoisse comme une sorte de stimulus de pensée, en tant qu'elle nous place devant le tout de l'être, au sens heideggérien, devant le tout de l'existence. C'est à elle qu'il convient de faire face et non à la mort elle-même. Il déplace donc le face à face réclamé par Hegel. "Der Mensch soll die Angst des Irdischen nicht von sich werfen; er soll in der Furcht des Todes - bleiben » (4). En effet, cette angoisse de mort est à sa façon, très singulière, une source de connaissance qui permet de rester dans la vie tout en sachant que cette dernière est marquée par la mort.

Il n'y a donc pas chez Rosenzweig de " pensée de la mort ». Le 26 novembre 1918, il écrit à Margrit Rosenstock: «Meinst du, ich hätte wohl ein klares Wort über den Tod geschrieben. Ich weiss es selbst nicht; er kommt immer wieder vor, immer wieder anders, aber nirgends mit endgültiger Klarheit $»^{14}$. Affirmation d'un non-savoir indépassable, non-savoir d'un Quelque-chose d'irréductible là où le geste spéculatif par excellence consisterait à faire ségaliser le savoir et le quelque-chose, d'une part, et le non-savoir et le néant d'autre part. Cette mise en non-correspondance de l'idéalisme avec soi est le stimulus de la destruction de la « philosophie » engagée dans la première partie du Stern, in philosophos. Elle

$14 \mathrm{~GB}, 200$. 
lui fournit son commencement, ce qu’on voit très bien dès la première phrase de l'Einleitung, laquelle connecte d'emblée deux mots, Tod et All, afin de s'acheminer à grande allure vers la description de la faillite de l'idéalisme unitotalisant. Dans une autre lettre, toujours adressée à Gritli, Rosenzweig écrit : «Der Tod ist ja überhaupt das Feste im Leben $»^{15}$. La mort est tout à la fois constante, fest, et protéiforme. Elle est " quelque-chose » de constant parce que chacun vit avec cette certitude, de façon " authentique " ou pas n'a guère d'importance ici. Cependant, elle nest pas réductible à une forme ou à une interprétation unique. Son statut n'est pas définissable pour tous, une fois pour toutes. Si tel était le cas, cela impliquerait d'une certaine manière qu'il est possible de l'apprivoiser, voire de se l'approprier, d' " apprendre à mourir » selon l'adage, ce qui est tout le contraire de ce que nous dit Rosenzweig.

Il y a donc, comme explique Schopenhauer dans le complément 41 déjà cité du Welt als Wille und Vorstellung, une relation consubstantielle, cooriginaire, entre la pensée, la raison, la philosophie et la conscience de notre statut mortel : « la connaissance lutte contre la crainte de la mort ». Le déni ou l'amortissement de ce quelque chose anime et donne son élan à l'idéalisme spéculatif (mais aussi aux religions) qui, sous couvert de regarder la mort droit dans les yeux, la réduit à rien, à un néant dont il convient de sortir par la voie de lêtre, comme au début de la Grande Logique hégélienne. L'Einleitung promet un autre chemin, celui de " la révolution post-hégélienne de la philosophie » (18), scandé et balisé par les trois noms mentionnés, Schopenhauer, Kierkegaard, Nietzsche, auxquels il faut ajouter le dernier Schelling. C'est bien ce chemin que Rosenzweig entend à son tour emprunter dans le Stern -mais à sa façon à lui.

Quelle est cette façon ? Elle consiste à prendre au sérieux, non point tant la mort, car celle-ci n’apporte pas de "clarté définitive ", que l'angoisse de la mort qui, elle, dit la vérité du Je fini. Quel en est l'effet le plus immédiat ? La réaffirmation d'un cri, le cri du Je vivant. Quel en est la conséquence intra-philosophique ? La détotalisation, la destruction, le bris de la totalité comme irrigués par les « bras de mort » qui s'y avancent. C'est à partir de ce bris que se dégagent comme d'eux-mêmes les "éléments "-et la philosophie élémentaire esquissée dans la première partie du Stern.

Le bénéfice secondaire de la " crainte de la mort » et de ses avatars idéalistes, c'est la connaissance du Tout. Pour Rosenzweig, c'est déjà quelque chose, cet Erkennen. Ni plus ni moins que le terme hégélien de l'histoire de la philosophie -une sorte de fin de la philosophie. Cette connaissance terminale, considérée depuis « la révolution post-hégélienne de la philosophie » ou depuis cette philosophie pour «notre temps » quest la "philosophie du point de vue », ne peut apparaître que biaisée, tronquée. C'est le premier volet du refus d’obtempérer (Gefolgschaft versagen, 22) à la philosophie du Tout, le second pouvant être rapporté au Cri. Articulé en deux pans, comme si le mensonge pouvait se décrire

\footnotetext{
${ }^{15}$ Lettre du 18 avril 1918, GB, 77.
} 
selon deux volets, logique et existentiel, il y a donc dans les vingt premières pages de l'Etoile, un refus très radical de tout Tout, pour ainsi dire. Refus gnoséologique et en fin de compte philosophique. Refus moral aussi -non pas en un sens axiologique, comme si l'on pourrait dériver ce refus du Tout de la raison pratique elle-même ou du Sujet transcendantal, à partir de quoi on déduirait des valeurs. Le Tout fait l'objet d'un refus et d'un bris, parce que le mensonge qu'il autorise est un vice existentiel, un mensonge du point de vue de l'existence, une inhumanité qui redouble une fausseté cognitive. L'Etoile commence où finit la philosophie et ses systèmes.

\section{Le maintien (Bewahrung) et l'avération (Bewährung)}

L'Einleitung met en place des axes méthodologiques et des procédures expérimentales qui président à la déconstruction élémentale mise en œuvre dans la première partie. Quatre outils sont expressément mentionnés par Rosenzweig, le Néant, le Oui, le Non, le Et. Ils servent à disposer deux grandes transversales épistémologiques, commandées l'une par le temps, l'autre par la parole, la seconde absorbant en quelque sorte la première.

Le premier rappel anti-idéaliste, c'est la différence entre le résultat et le commencement, appuyée sur les métaphores de la valise dans le Stern (125) et du programme de théâtre dans Das neue Denken ${ }^{16}$ : ce qui est présent dans la pensée, c'est-à-dire dans le temps du concept (dont Hegel assurait qu'il se donnait comme une sorte de maintenant éternel), n'est que le résultat d'un passé dans le réel. Et ce qui est résultat doit être ce qui met en branle la pensée, son re-commencement en quelque sorte après le bris du tout. Dieu-MondeHomme, obtenu par la détotalisation de l'unitotalité, se tiendra aussi bien au principe de la nouvelle pensée en tant que « réponse à la vieille question philosophique de la quoddité ${ }^{17}$. Le Stern se donne pour tâche d'en construire les " éléments » en en déconstruisant la structure : les trois monismes, les trois substances, les trois points, etc. On comprend pourquoi Rosenzweig a pu donner à ses lecteurs le conseil (à ne pas suivre aveuglément !) de passer très vite sur la première partie : le bris, les trois substances fixes, la ré-initialisation de la pensée, les figures du «tout» (excluante, incluante), etc. -toutes choses qui en passent par la temporalisation du connaître. Or tout commence après le « commencement » du Stern, après le prémonde, dans le monde, lorsqu'il s'agit de " révéler » pourquoi cette structure élémentale elle-même, issue du bris du tout, pro-vient d'une expérience, d'une épreuve, d'un Erlebnis. En effet, dans la première partie, on est toujours dans la pensée, fût-elle "nouvelle ", on est toujours dans le prémonde, mais plus du tout de la même façon : ce prémonde ne se prend pas pour le monde!

\footnotetext{
16 GS III, 147.

17 Ibid., 148.
} 
A ce rappel de la "philosophie " au temps et par le temps s'adjoint pour le surdéterminer une distinction fondamentale qui se trouve à la page $16:$ "Die Wahrheit bewährt nicht die Wirklichkeit, sondern die Wirklichkeit bewahrt die Wahrheit. Das Wesen der Welt ist diese Bewahrung (nicht Bewährung) der Wahrheit ». Il convient donc pour einen gesunden Menschenverstand de ne jamais confondre la preuve et la garde, la vérité et l'effectivité -le mur et l'image. Tels sont les enjeux portés par « die Methode, die in der Vorsilbe " meta » bezeichnet ist» (21). La méthode méta permet en effet de comprendre (et ceci, de provenance schellingienne, est essentiel pour lire le Stern) que c'est bel et bien l'effectivité qui permet la garde de l'essence de la vérité. La raison, le concept sont supportés par l'effectivité qui en assure le maintien en quelque sorte. La maintenance du vrai s'effectue par ce qui est plus vaste que le vrai, comme le mur pour le tableau qui y est suspendu. La « nouvelle pensée » doit inventer un nouveau type d'exercice " philosophique ", une nouvelle méthode qui ne reconduise plus la Wirklichkeit à son essence par réduction et fondation mais qui en discernera la vérité dans la parole en tant qu'antonyme du concept. Ce Sprachdenken procède d'une défection de l'être. Tel est le sens de la formule de Das neue Denken : "das Wirkliche 'ist' nicht ${ }^{18}$. Ce qui, évidemment, ne veut pas dire : l'effectif n'existe pas, mais il n'est rien d’étant, il échappe à toute essence, à toute quiddité. "L'effectif n'est pas " signifie dire que lêtre n'est pas effectif. Le principe énoncé à la page 16 du Stern croise évidemment la monstration de Das neue Denken. On peut d'ailleurs considérer que la révélation est une façon de produire une réversion de l'être en effectivité, du ist (la Ist-frage comme question de la philosophie pérenne) en wirkt (la Wirklichkeit comme garde et maintien).

L'impératif, dans notre Einleitung, est de sortir de la feinte logique et du leurre existentiel du "Tout » total, ce piège à deux mâchoires. Rosenzweig y engage une démarche spécifique qui permettra de " détricoter » la totalité spéculative (19). Au terme du Bris qui sopère au long des chapitres de la première partie, on obtiendra quelque chose de positif, comme si cet envol hors du néant-et-du-tout permettait d'accéder à un autre genre de connaissance que la connaissance une et universelle, à la fois plus fragile, car le tout est le non-vrai, et plus assurée, puisque le vrai est le non-tout. Après le Bris, nous aurons donc entre les mains des "parties ", des morceaux, des éléments, le Dieu mythique, le Monde plastique, l'Homme tragique -qui nous apparaîtront alors comme des "pures hypothèses" (91) et comme des touts. Mais ces touts hypothétiques ne se retotaliseront plus car ils ne sont pas des Touts, même s'ils peuvent bien en avoir l'apparence " moniste ». Le Tout est brisé, non seulement en trois mais en une multiplicité miroitante de facticités (95). Le Bris donne lieu à un emboîtement de bris, à une élémentarisation continuée des éléments. En effet, chacun de ces éléments, chaque quelque-chose a l'ambition récurrente de se reconstituer en Tout, en une "Riesenform» (95) gigantesque et monstrueuse. A

${ }^{18}$ GS III, 148. 
partir de là, le geste philosophique quasi-idiosyncrasique de Rosenzweig dans le Stern consistera à toujours multiplier ce qui se donne dans l'unicité d'une forme stable tenue en suspicion, à soustraire l'Un, à diviser le Tout. L'hubris du Tout et de la totalisation fait du bris une tâche sans terme, et du " philosopher après Hegel » un ouvrage incessant plus qu'un programme accompli. Ce qui donc restera après le bris, après chaque bris, ce sera de la relation, de la circulation, de la liaison et de la reliaison, une diachronie qui n'est rien d'autre que la temporalité du sujet existant. Du fragile et du brisé -mais cette fragilité détient désormais une force singulière, la force de l'ouvert, l'ouverture par et dans la langue. Car les éléments du Tout ont toujours déjà vocation à se mettre à parler (121), leurs contenus étant autrement inassemblables (154). Cet assemblage par la parole (et par le temps et par l'autre, comme le rappellera Das neue Denken) ne pourra plus jamais relever d'une totalisation. Le contenu des éléments est absolument hors d'état de s'assembler en totalité car, dans leur surgissement, les éléments retiennent encore des contenus qui ne peuvent entrer en action que dans d'autres directions ( der ganze Inhalt der Elemente kann gar nicht in einem einzelnen Begriff zusammentreten », 154).

Le mensonge brisé, demeurent ainsi des morceaux d'existence, du temps, des temps (Création, Révélation, Rédemption), des langues, des langues silencieuses et des langues sonores. Là, l'Einleitung s'ouvre vers l'ensemble des trois parties qui composent le Stern, comme un fleuve se jette dans une mer incertaine.

\section{lectures supplémentaires}

Bertolino, L., Das Nichts und die Philosophie. Rosenzweig zwischen Idealismus und einer Hermeneutik der religiösen Erfahrung, in: Wolfdietrich Schmied/ Kowarzik (Hg.), Franz Rosenzweigs »neues Denken«. Internationaler Kongreß Kassel 2004, 2 Bde, Karl Alber, Freiburg/München, 2006, Bd. I: Selbstbegrenzendes Denken - in philosophos, 111-125 [https://philarchive.org /archive/BERDNU]

Pollock, B. (2009). Franz Rosenzweig and the systematic task of philosophy. Cambridge University Press. 